\title{
Voxel-wise correlations between cognition and cerebral blood flow using arterial spin- labeled perfusion MRI in patients with Alzheimer's disease: a cross-sectional study
}

Tomohiro Kaneta ${ }^{1 *}$, Omi Katsuse ${ }^{2}$, Takamasa Hirano ${ }^{1}$, Matsuyoshi Ogawa' ${ }^{1}$ Ayako Shihikura-Hino ${ }^{1}$, Keisuke Yoshida', Toshinari Odawara ${ }^{2}$, Yoshio Hirayasu ${ }^{2}$ and Tomio Inoue ${ }^{1}$

\begin{abstract}
Background: To analyze voxel-wise correlation between cerebral blood flow (CBF) measured using ASL-MRI and cognition in patients with Alzheimer's disease (AD).

Methods: Forty-one patients diagnosed with $A D$ or mild cognitive impairment due to AD were recruited for this study. CBF images were obtained using ASL-MRI $(n=41)$ with a post-labeling delay (PLD) of 1.5 and $2.5 \mathrm{~s}\left(\mathrm{PLD}_{1.5}\right.$ and $\mathrm{PLD}_{2.5}$, respectively) using a $3 \mathrm{~T}$ scanner, in addition to brain perfusion SPECT with $\mathrm{N}$-isopropyl-4-[I123]iodoamphetamine $(n=28)$. Voxel-based analyses were performed for ASL-MRI and SPECT using MiniMental State Examination (MMSE) scores as covariates. Differences in CBF between PLD 1.5 and $\mathrm{PLD}_{2.5}$ were assessed using a paired t-test with SPM12.
\end{abstract}

Results: Significant positive correlations were observed between MMSE scores and CBF at PLD 1.5 in the right posterior cingulate cortex (PCC), and both temporo-parietal association cortexes. At $\mathrm{PLD}_{2.5}$, significant positive correlations were determined for MMSE scores and CBF in the superior parietal lobule and the right temporoparietal association cortex. SPECT showed significant positive correlations in the PCC and both temporoparietal association cortexes (right-side dominant). PLD 1.5 showed significantly higher CBF than $\mathrm{PLD}_{2.5}$ in the proximal areas of vascular territories of the anterior, middle, and posterior cerebral arteries.

Conclusions: Significant positive correlations in CBF, measured with both ASL-MRI and SPECT, with cognition were found in the PCC and temporo-parietal association cortexes. PLD 1.5 and PLD $_{2.5}$ showed similar correlations with cognition, although the CBF images had significant differences.

\section{Background}

For the evaluation of cerebral blood flow $(\mathrm{CBF})$ in patients with Alzheimer's disease (AD), brain perfusion single photon emission computed tomography (SPECT) has been widely used for both visual and voxel-based analysis [1-6]. More recently, arterial spin labeling magnetic resonance imaging (ASL-MRI) has also been applied to evaluate CBF. This approach is a noninvasive technique that uses magnetically labeled water in arterial

\footnotetext{
* Correspondence: kaneta@yokohama-cu.ac.jp

${ }^{1}$ Department of Radiology, Yokohama City University, 3-9 Fukuura,

Kanazawa-ku, Yokohama 236-0004, Japan

Full list of author information is available at the end of the article
}

blood as an endogenous contrast medium [6-9]. Using this method to make comparisons between CBF in patients with various forms of dementia to demographically matched healthy controls has yielded significant findings. Both $\mathrm{AD}$ and mild cognitive impairment (MCI) have been associated with decreased CBF in the middle occipital areas, medial temporal lobe, and even more significantly in the parietal lobe [10]. Similar decreases in $\mathrm{CBF}$ have been reported in the posterior cingulate and precuneus, in addition to the frontal and parietal regions $[11,12]$. ASL-MRI has also been successfully applied for the differential diagnosis of dementia [13, 14], and showed a high degree of concordance with FDG PET 
diagnoses $[15,16]$. However, at present, few studies have reported voxel-wise correlations between CBF measured by ASL-MRI and cognition in patients with AD. The detection of regions with significant correlations between $\mathrm{CBF}$ and cognition may lend validity to the idea that regional CBF can serve as a biomarker of the neural changes underlying cognitive decline.

Among several ASL labeling approaches, pseudocontinuous ASL (PCASL) is now mainly used. This is a modification of continuous labeling in which a long train of short pulses is used to achieve flow-induced adiabatic inversion of arterial blood water, and is recommended for clinical imaging by the International Society for Magnetic Resonance in Medicine (ISMRM) study group on perfusion imaging [17]. Arterial water at the base of the brain is labeled by the PCASL pulses, and the brain is imaged after a fixed time interval. This is termed postlabeling delay (PLD) and is a key parameter for ASL imaging. Several values of PLD such as $1.5,2.0$, and $2.5 \mathrm{~s}$ have been used in the studies of AD [18-20]. Generally, PLD is fixed to one value, but using multiple PLDs seems interesting to evaluate the optimal PLD and the difference between varying PLDs. In addition, the influence of different PLDs on the results of voxel-wise analyses has not been evaluated.

In this study, we evaluated and compared the correlation between cognition and CBF measured by ASLMRI with a PLD of $1.5 \mathrm{~s}$ and $2.5 \mathrm{~s}\left(\mathrm{PLD}_{1.5}\right.$ and $\mathrm{PLD}_{2.5}$, respectively) in patients with $\mathrm{AD}$. We also compared the results with brain perfusion SPECT.

\section{Methods}

\section{Subjects}

Forty-one patients who underwent MRI with diagnosis of AD or mild cognitive impairment (MCI) due to AD between September 2015 and March 2016 were recruited. Patients with $\mathrm{AD}$ were included if they met the criteria for probable $\mathrm{AD}$ established by the $\mathrm{Na}$ tional Institute of Neurological and Communicative Disorders and Stroke-Alzheimer Disease and Related Disorders Association (NINCDS/ADRDA) [21]. Patients were excluded from the study if they had a significant history of psychiatric or neurological disorders other than AD, including stroke, head injury, epilepsy, psychiatric disorders, alcohol abuse, or other serious medical conditions. All patients underwent MR scanning at $3.0 \mathrm{~T}$ and standard dementia screening, which included a medical history check, Mini-Mental State Examination (MMSE), neuropsychological testing, and MR imaging. Cognition was assessed using the MMSE, which evaluates general cognitive function, including orientation to time and place, attention and calculation, language, and memory [22]. Among these, 28 patients also underwent brain perfusion SPECT using $N$-isopropyl-4$\left[{ }^{123} \mathrm{I}\right]$ iodoamphetamine $\left({ }^{123} \mathrm{I}-\mathrm{IMP}\right)$ within 1 month of MR scanning at our hospital. This tracer has been reported to be feasible for the quantitative evaluation of $\mathrm{CBF}$ in routine clinical practice [23]. Most of others underwent SPECT using other tracer or at another hospital, but we did not include their SPECT images for the analysis in this study. We used all available images of both ASL and SPECT obtained from all patients.

\section{MR imaging}

Images were acquired using a 3.0 T MR system (Discovery750w, GE Medical Systems) and a 12-channel head coil.

Anatomic information was obtained from a sagittal three-dimensional (3D) T1-weighted sequence, (the parameters include: $\mathrm{TR}=6.6 \mathrm{~ms}$, $\mathrm{TE}=2 \mathrm{~ms}, 14^{\circ} \mathrm{flip}$ angle, matrix $=256 \times 256,170$ stions, voxel size $=1.0 \times 0.9 \times 0.9 \mathrm{~mm}^{3}$, FOV $=23 \times 23 \mathrm{~cm}$ ), acquisition time was $6 \mathrm{~min} 0 \mathrm{sec}$.

The ASL sequence consisted of a 3D, multi-delay PCASL, with a fast spin-echo acquisition with background suppression. The labeling plane was set at the base of the brain without the information of MR angiography. The imaging protocol of $\mathrm{PLD}_{1.5}$ was as follows: $\mathrm{TR}=4641 \mathrm{~ms}, \mathrm{TE}=10.7 \mathrm{~ms}$, locations $=36$, FOV $=23 \times 23 \mathrm{~cm}$, voxel size $=2 \times 2 \times 4 \mathrm{~mm}^{3}$, PLD = $1.5 \mathrm{~s}$, labeling duration $=1.5 \mathrm{~s}$, number of excitations $(\mathrm{NEX})=1$, acquisition time was $1 \mathrm{~min} 33 \mathrm{~s}$. The imaging protocol of $\mathrm{PLD}_{2.5}$ was as follows: $\mathrm{TR}=5336 \mathrm{~ms}$, $\mathrm{TE}=10.7 \mathrm{~ms}, \mathrm{PLD}=2.5 \mathrm{~s}, \mathrm{NEX}=2$, all other parameters maintained the same, and the acquisition time was $2 \mathrm{~min} 51 \mathrm{~s}$.

A two-compartment model with finite labeling duration was used for PCASL quantification. An approximately proton-density-weighted image was obtained by turning the labeling RF off. Calculation of flow was based on the following equation.

$$
\mathrm{f}=\frac{\lambda\left(S_{c t r l}-S_{l b l}\right)\left(1-e^{-\frac{t_{s a t}}{T_{1 g}}}\right)}{2 \alpha T_{1 b}\left(1-e^{-\frac{\tau}{T_{1 b}}}\right) S_{r e f}} e^{\frac{w}{T_{1 b}}}
$$

where $f$ is the measured CBF; $S$ is the signal from the control, label, or reference image as determined by the subscript, $S_{\mathrm{lbl}}$ is the label image, i.e. image obtained with unbalanced RF labeling that gives rise to perfusion weighting, $S_{\text {ctrl }}$ is the control image, i.e. image obtained with balanced RF so that the arterial blood is not labeled, $S_{\text {ref }}$ is the proton density image that is obtained with labeling RF turned off; $T_{1} b$ is the T1 of blood; $\mathrm{T}_{1} g$ is the T1 of gray matter; $\alpha$ is the labeling efficiency; $\lambda$ is the brain-blood partition coefficient; $t_{\text {sat }}$ is the saturation time for proton density images (2 s); $\tau$ is the 
labeling duration $(1.5 \mathrm{~s})$; and $\omega$ is the post label delay. We used a gray matter T1 estimate of $1.2 \mathrm{~s}$ and an assumed blood T1 of $1.6 \mathrm{~s}$ [24]. The labeling efficiency was assumed to be 0.8 for the PCASL.

\section{Preprocessing and MR imaging data analysis}

Data analyses were carried out by using Statistical parametric mapping 12 (SPM12) (http://www.fil.ion.ucl.ac.uk/ spm/software/spm12/). Both 3D T1-weighted and 3D PCASL images were corrected for image distortion due to gradient non-linearity using 'GradWarp' [25]. Preprocessing of 3D T1-weighted images consisted of realignment, coregistration, and segmentation.

ASL images were linearly registered to the brain extracted from the 3D T1-weighted images. Mean wholebrain $\mathrm{CBF}$ values were calculated in the brain mask, converted to quantitative CBF maps in the unit of $\mathrm{mL} /$ $100 \mathrm{~g} / \mathrm{min}$, spatially normalized to the Montreal Neurological Institute (MNI) space with a 2-mm isotropic resolution, and smoothed with an isotropic kernel of $6 \mathrm{~mm}$. Complementary voxel-wise comparisons of 2 kinds of CBF maps were performed by SPM12 as well. Correlation results were statistically thresholded at $p<0.001$ uncorrected. All results were shown in the MNI space. The locations with significant results were determined using the "Neuromorphometrics" function of SPM12.

\section{Brain perfusion SPECT}

SPECT was performed by intravenous injection of $148 \mathrm{MBq}{ }^{123}$ I-IMP (Nihon Mediphysics, Hyogo, Japan) in participants seated at rest with their eyes open. A dual-head gamma camera with integrated thin-slice diagnostic CT (Symbia ${ }^{\circ}$ T16, Siemens Healthcare, Molecular Imaging, Hoffman Estates, IL, USA) was used. The SPECT scans were acquired using low-mediumenergy general-purpose collimation, a $128 \times 128$ matrix of $3.3-\mathrm{mm}$ pixel size, and a total of $300 \mathrm{~s} /$ rotation in a continuous-rotation mode. Subsequent to the SPECT acquisition, a reduced-dose CT scan was acquired with $130 \mathrm{kV}$ and 150 ref. mAs. The CT data were generated with a 3-mm slice thickness using a smooth reconstruction kernel (H08s, Siemens Healthcare) and a 2-mm slice thickness using a medium kernel (H31s medium sharp, Siemens Healthcare).

SPECT reconstruction was performed using filtered back projection using a Butterworth filter with cutoff $=0.35 / \mathrm{cm}$ and order 8 . A uniform attenuation correction was performed using Chang's method with $\mu=0.11$.

\section{Statistical analysis}

Voxel-based analyses were performed for $\mathrm{PLD}_{1.5}, \mathrm{PLD}_{2.5}$ and brain perfusion SPECT using MMSE scores as covariates. Differences of CBF between $\mathrm{PLD}_{1.5}$ and $\mathrm{PLD}_{2.5}$ were assessed by paired $\mathrm{t}$-test. The individual brain images were normalized by the global values. The $p$ value threshold was 0.001 at the voxel level, and the regions with the extent under the expected voxels per cluster were omitted. The plots of the correlation between the voxel values and MMSE scores at the most significant area are provided. These were performed by SPM12.

\section{Results}

\section{Patient demographics}

Patient demographics, including age, sex, and MMSE scores, are summarized in in Table 1. There were no significant differences between the total population $(n=41)$ and the subpopulation that underwent SPECT $(n=28)$.

\section{Voxel-wise correlations between CBF and cognition}

Using ASL-MRI, PLD ${ }_{1.5}$ scans revealed significant positive correlations with MMSE results in the right posterior cingulate cortex (PCC) and both temporo-parietal association cortexes, in addition to the right rectal gyrus (Fig 1a). PLD 2.5 showed significant positive correlations in the superior parietal lobule but not PCC, and the right temporo-parietal association cortex only. Positive correlations for the right inferior temporal lobule and right fusiform gyrus were also significant (Fig 2a). Brain perfusion SPECT identified significant positive correlations in the PCC and both temporo-parietal association cortexes (right-side dominant), and the left fusiform gyrus (Fig 3a). The plots of the correlation between the voxel values and MMSE scores at the most significant area are shown in Figs. 1b, 2b, and 3b. All of them showed linear relationships. The expected voxels per cluster for $\mathrm{PLD}_{1.5}, \mathrm{PLD}_{2.5}$, and SPECT were 63.4, 40.8, and 60.9, respectively. Cluster-level statistics for all rendered clusters are summarized in Table 2 .

\section{Voxel-wise differences in CBF between $\mathrm{PLD}_{1.5}$ and $\mathrm{PLD}_{2.5}$} $\mathrm{PLD}_{1.5}$ showed significantly higher CBF than $\mathrm{PLD}_{2.5}$ at the proximal areas of vascular territories of the anterior, middle, and posterior cerebral arteries (Fig 4).

Table 1 Demographic and neuropsychological summary of subjects included in the current study ${ }^{\text {a }}$

\begin{tabular}{lll}
\hline & Total & SPECT \\
\hline Age, $y$ & $79.0(6.4)$ & $79.5(6.6)$ \\
No. of patients & 41 & 28 \\
Gender, M/F & $17 / 24$ & $9 / 19$ \\
MMSE & $23.0(3.8)$ & $23.0(3.9)$ \\
\hline
\end{tabular}

${ }^{\mathrm{a}}$ Data are presented as mean (SD). 


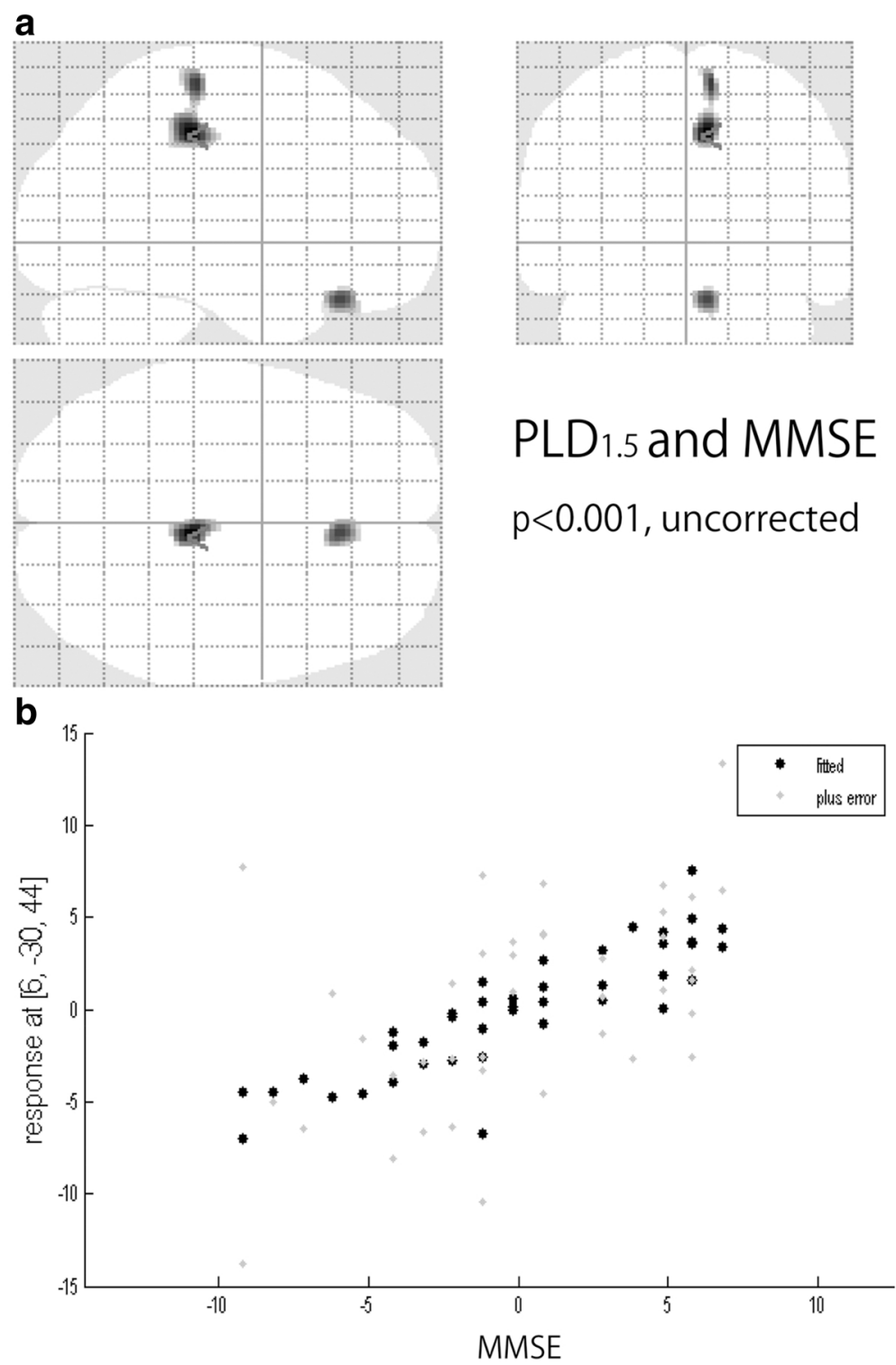

Fig. 1 a Voxel-wise correlations between cognition measured with MMSE and CBF measured using $\operatorname{PLD}_{1.5}(p<0.001, T=3.32$, uncorrected). $\mathbf{b}$ The plots of the correlation between the voxel values and MMSE scores at the most significant area (right posterior cingulate cortex)

\section{Discussion}

From the results of our study, we identified significant positive correlations between $\mathrm{CBF}$ and cognition, when measuring using both ASL-MRI and SPECT. The main areas with significant results were located in PCC and temporo-parietal association cortexes, which are known to show decreases in perfusion or metabolism during cognitive decline in association with AD [25-28]. These areas are the posterior parts of the default mode network (DMN), which has a primary network center in the PCC. These regions have a strong functional connection to the left and right inferior parietal lobule (IPL), ventral and dorsal medial prefrontal cortex, and lateral temporal lobes [29]. Functional MRI studies have also consistently implicated the DMN as the most vulnerable network in $\mathrm{AD}[30,31]$. The posterior (temporo-parietal-predominant) DMN may be particularly susceptible in early-stage AD [32-34]. These studies therefore support our findings that a significant correlation exists between $\mathrm{CBF}$ and cognition, in the PCC and IPL. To the best of our knowledge, ours is the first report that succeeded in demonstrating such correlations between $\mathrm{CBF}$ and cognition using ASL-MRI, with supporting data using SPECT. These correlations may support the idea that regional CBF can serve as a biomarker of the neural changes underlying cognitive decline. As shown in Figs. 
a

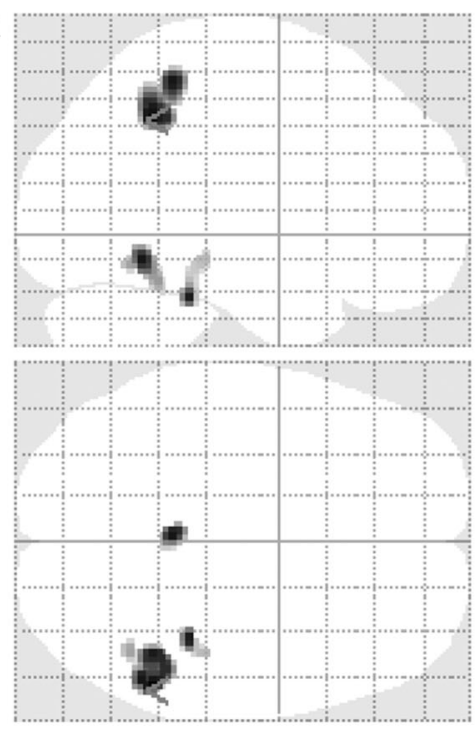

b

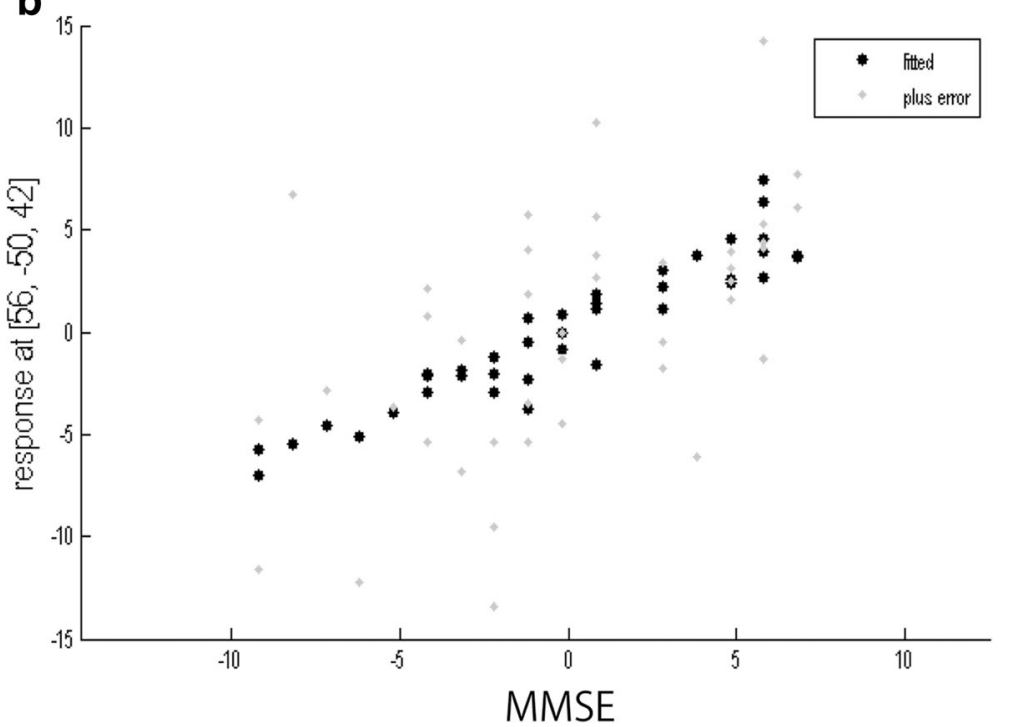

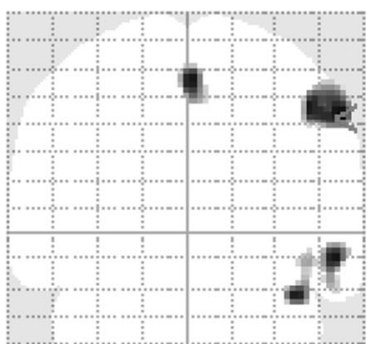

\section{PLD2.5 and MMSE}

$p<0.001$, uncorrected

Fig. 2 a Voxel-wise correlations between cognition measured with MMSE and CBF measured using $\operatorname{PLD}_{2.5}(p<0.001, T=3.32$, uncorrected). b The plots of the correlation between the voxel values and MMSE scores at the most significant area (right inferior parietal lobule)

$1 \mathrm{~b}$ and $2 \mathrm{~b}$, the voxel values of SPM results dropped linearly with decreasing MMSE scores. Our results also showed similar significant correlations between cognition and CBF using ASL-MRI with PLD 1.5 and PLD $_{2.5}$, and SPECT. The measurement of CBF is thought to be influenced by modalities, tracers, and parameters. In fact, our results showed that ASL-MRI with PLD $_{1.5}$ and $\mathrm{PLD}_{2.5}$ have significant differences. A significantly higher CBF was found at the adjacent areas of anterior cerebral arteries, middle cerebral arteries and posterior cerebral arteries for $\mathrm{PLD}_{1.5}$ compared to $\mathrm{PLD}_{2.5}$, suggesting early perfused areas. Liu et al. [35] evaluated the $\mathrm{CBF}$ of $\mathrm{AD}$ patients using ASL-MRI 
a

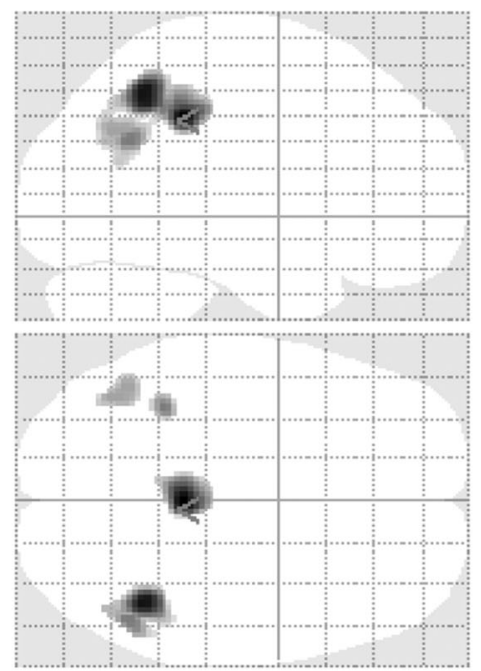

b
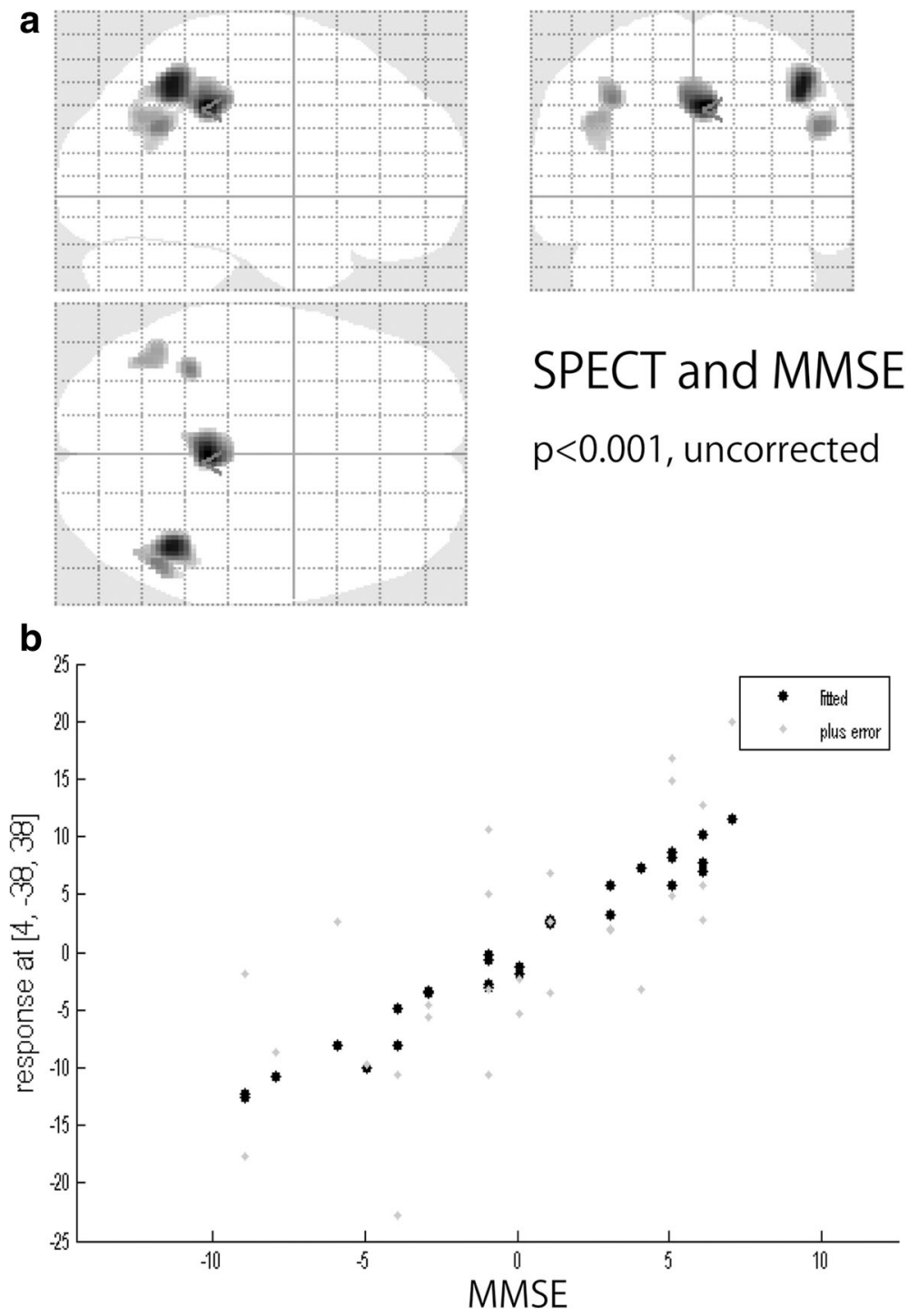

\section{SPECT and MMSE}

\section{$\mathrm{p}<0.001$, uncorrected}

Fig. 3 a Voxel-wise correlations between cognition measured with MMSE and CBF measured using brain perfusion SPECT ( $p<0.001, T=3.45$, uncorrected). $\mathbf{b}$ The plots of the correlation between the voxel values and MMSE scores at the most significant area (right posterior cingulate cortex)

imaging system (eZIS)" [37, 38] have been commonly used for the individual diagnoses in daily practices. Such voxel-wise methods may be helpful for making individual diagnoses using ASL-MRI. However, an age-specific normal database is required for the detection of significant abnormalities of individual images.

Our results also demonstrate a significant correlation between $\mathrm{CBF}$ and cognition at the right rectal gyrus for $\mathrm{PLD}_{1.5}$, and the right inferior temporal lobule and fusiform gyrus for $\mathrm{PLD}_{2.5}$. However, these areas are located at the edge of the brain, and these results might be caused by the errors during anatomical standardization and/or masking, and seem to be artifacts.

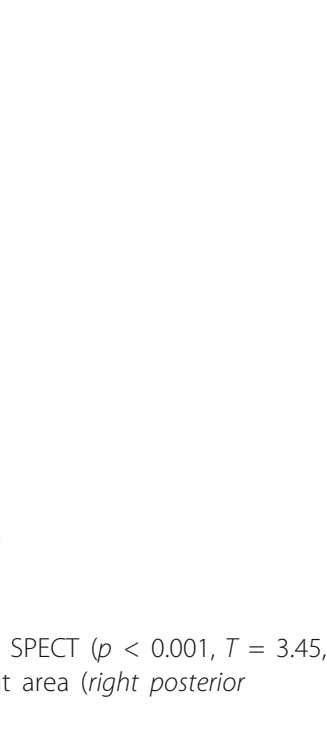


Table 2 Summary of voxel-wise CBF correlations with MMSE

\begin{tabular}{|c|c|c|c|c|c|c|c|}
\hline Modality & Anatomic label & $x$ & $\mathrm{y}$ & z & t statistic & z score & Voxels \\
\hline \multirow[t]{2}{*}{$\mathrm{PLD}_{1.5}$} & R PCC & 6 & -30 & 44 & 4.65 & 4.11 & 209 \\
\hline & $\mathrm{R}$ gyrus rectus & 6 & 30 & -26 & 4.20 & 3.79 & 97 \\
\hline \multirow[t]{4}{*}{$P L D_{2.5}$} & $\mathrm{RIPL}$ & 56 & -50 & -42 & 4.30 & 3.85 & 258 \\
\hline & $\mathrm{R}$ inferior temporal & 54 & -54 & -12 & 4.20 & 3.78 & 82 \\
\hline & SPL & 0 & -42 & 56 & 4.20 & 3.78 & 80 \\
\hline & R fusiform gyrus & 38 & -36 & -26 & 4.17 & 3.76 & 45 \\
\hline \multirow[t]{5}{*}{ SPECT } & R PCC & 4 & -38 & 38 & 5.05 & 4.15 & 361 \\
\hline & R PGp (IPL) & 44 & -54 & 48 & 4.93 & 4.08 & 341 \\
\hline & L PGa (IPL) & -44 & -60 & 32 & 3.85 & 3.38 & 163 \\
\hline & R PGa (IPL) & 50 & -58 & 28 & 4.15 & 3.58 & 95 \\
\hline & L inferior parietal & -36 & -46 & 42 & 4.13 & 3.57 & 90 \\
\hline
\end{tabular}

Voxel coordinates represent the peak voxel in local maxima, coordinates are expressed in Montreal Neurological Institute stereotactic space. $P<0.001$, uncorrected. The regions with the extent under the expected voxels per cluster were omitted

$R$ right, L left, $P C C$ posterior cingulate cortex, IPS intraparietal sulcus, IPL inferior parietal lobule, SPL superior parietal lobule, PGa anterior angular gyrus, PGp: posterior angular gyrus

study. Furthermore, no conclusions could be drawn on the individual subject level, as the analyses were all performed on pooled data from each group.

\section{Conclusions}

Significant positive correlations between $\mathrm{CBF}$ and cognition were detected using ASL-MRI with PLD $_{1.5}$ and $\mathrm{PLD}_{2.5}$ in the PCC and temporo-parietal association cortexes. These results were supported by brain perfusion SPECT. CBF in these areas decreased with increasing severity of cognitive impairment. These measures could therefore be important in the clinical diagnosis and evaluation of AD. The difference of PLD made significant differences in the measured CBF, but not in the voxel-wise correlation with cognition.
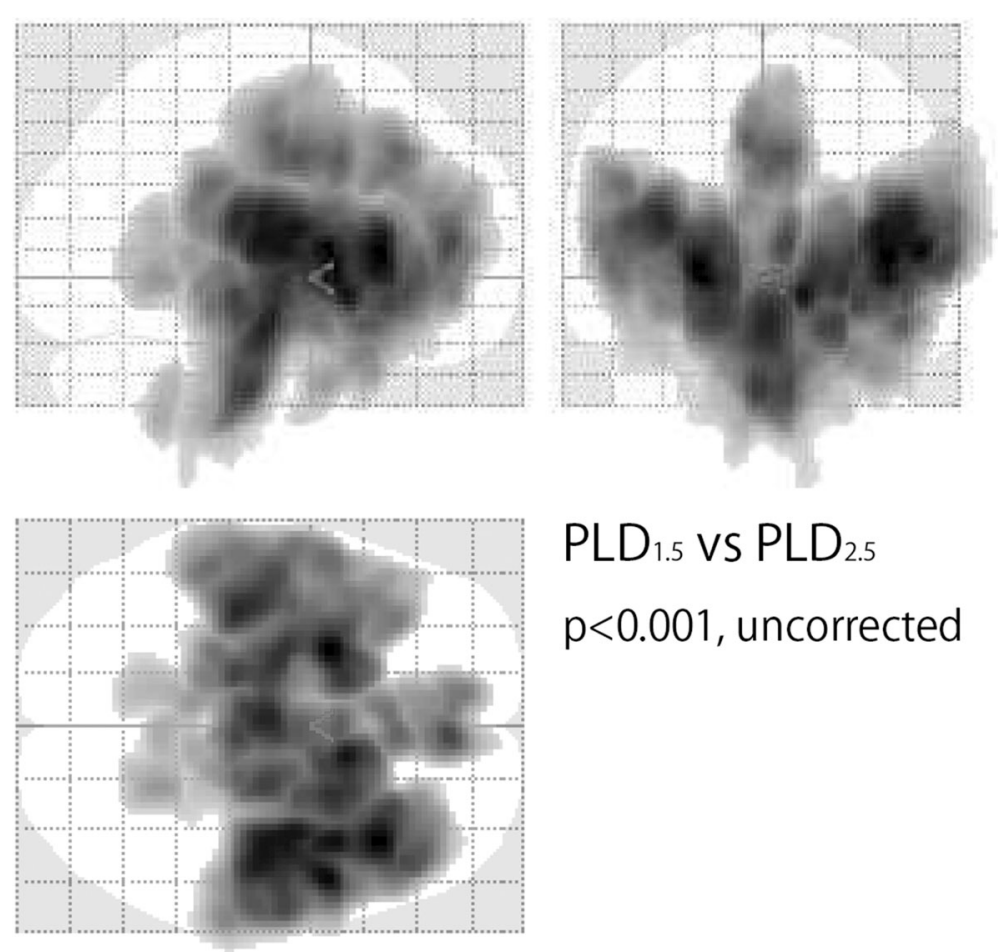

\section{PLD $_{1.5}$ Vs PLD 2.5}

$p<0.001$, uncorrected

Fig. 4 Voxel-wise CBF differences between $\mathrm{PLD}_{1.5}$ and $\mathrm{PLD}_{2.5}$ by paired t-test. $\mathrm{PLD}_{1.5}$ showed significantly higher $C B F$ than $P L D_{2.5}$ in the proximal areas of vascular territories of the anterior, middle, and posterior cerebral arteries $(p<0.001, T=3.32$, uncorrected) 


\section{Acknowledgements}

We thank Mr. Yuki Koyo for his assistance with data collection and analyses, and Mr. Naoki Hirata for his advice for image processing of ASL-MRI.

\section{Funding}

The study has not been supported by any grants.

\section{Availability of data and materials}

All relevant data are presented in the manuscript. Our raw data are coded and the key is available only to the responsible researchers. The Ethics Committee at Yokohama City University has not given permission to let anyone else deal with the data. If requested, by contacting the corresponding author, Tomohiro Kaneta, we can give more information concerning the background of our statistical computations.

\section{Authors' contributions}

TK, OK, TO, YH and TI conceived and designed the study. TK obtained ethical approval. $\mathrm{TH}$ and $\mathrm{MO}$ synthesized and analyzed images. $\mathrm{AH}$ and $\mathrm{KY}$ collected the data and performed the statistical analysis. TK wrote the article with support from YH and TI. OK and TO revised the article. All authors read and approved the final manuscript.

\section{Competing interests}

The authors declare that they have no competing interests.

\section{Consent for publication}

Not applicable.

\section{Ethics approval and consent to participate}

All subjects provided written informed consent to participate the clinical research and to publish papers. The principles of the Declaration of Helsinki were followed and approval was obtained by the ethics committee of Yokohama City University (B160500004).

\section{Publisher's Note}

Springer Nature remains neutral with regard to jurisdictional claims in published maps and institutional affiliations.

\section{Author details}

'Department of Radiology, Yokohama City University, 3-9 Fukuura, Kanazawa-ku, Yokohama 236-0004, Japan. ${ }^{2}$ Department of Psychiatry, Yokohama City University, 3-9 Fukuura, Kanazawa-ku, Yokohama 236-0004, Japan.

Received: 8 December 2016 Accepted: 4 May 2017

\section{Published online: 15 May 2017}

\section{References}

1. Jagust $W$, Thisted R, Devous MD Sr, et al. SPECT perfusion imaging in the diagnosis of Alzheimer's disease: a clinical-pathologic study. Neurology. 2001;56:950-6.

2. Bonte FJ, Weiner MF, Bigio EH, White $C L$ 3rd. Brain blood flow in the dementias: SPECT with histopathologic correlation in 54 patients. Radiology. 1997;202:793-7.

3. Matsuda $\mathrm{H}$. The role of neuroimaging in mild cognitive impairment. Neuropathology. 2007;27:570-7.

4. Matsuda $\mathrm{H}$. Role of neuroimaging in Alzheimer's disease, with emphasis on brain perfusion SPECT. J Nucl Med. 2007:48:1289-300

5. Imabayashi E, Matsuda H, Asada T, et al. Superiority of 3-dimensional stereotactic surface projection analysis over visual inspection in discrimination of patients with very early Alzheimer's disease from controls using brain perfusion SPECT. J Nucl Med. 2004;45:1450-7.

6. Tang BN, Minoshima S, George J, et al. Diagnosis of suspected Alzheimer's disease is improved by automated analysis of regional cerebral blood flow. Eur J Nucl Med Mol Imaging. 2004;31:1487-94

7. Dai W, Lopez OL, Carmichael OT, Becker JT, Kculler LH, Gach HM. Mild cognitive impairment and Alzheimer disease: patterns of altered cerebral blood flow at MR imaging. Radiology. 2009:250:856-66.

8. Chao LL, Pa J, Duarte A, et al. Patterns of cerebral hypoperfusion in amnestic and dysexecutive MCl. Alzheimer Dis Assoc Disord. 2009:23: 245-52.
9. Johnson NA, Jahng GH, Weiner MW, et al. Pattern of cerebral hypoperfusion in Alzheimer disease and mild cognitive impairment measured with arterial spin-labeling MR imaging: initial experience. Radiology. 2005;234:851-9.

10. Asllani I, Habeck C, Scarmeas N, Borogovac A, Brown TR, Stern Y. Multivariate and univariate analysis of continuous arterial spin labeling perfusion MRI in Alzheimer's disease. J Cereb Blood Flow Metab. 2008:28:725-36.

11. Alexopoulos P, Sorg C, Förschler A, et al. Perfusion abnormalities in mild cognitive impairment and mild dementia in Alzheimer's disease measured by pulsed arterial spin labeling MRI. Eur Arch Psychiatry Clin Neurosci. 2012; 262:69-77.

12. Yoshiura T, Hiwatashi A, Noguchi T, et al. Arterial spin labelling at 3-T MR imaging for detection of individuals with Alzheimer's disease. Eur Radiol. 2009:19:2819-25.

13. Mak HK, Chan Q, Zhang Z, et al. Quantitative assessment of cerebral hemodynamic parameters by QUASAR arterial spin labeling in Alzheimer's disease and cognitively normal elderly adults at 3-tesla. J Alzheimers Dis. 2012;31:33-44

14. Du AT, Jahng GH, Hayasaka S, et al. Hypoperfusion in frontotemporal dementia and Alzheimer disease by arterial spin labeling MRI. Neurology. 2006;67:1215-20.

15. Steketee RM, Bron EE, Meijboom R, et al. Early-stage differentiation between presenile Alzheimer's disease and frontotemporal dementia using arterial spin labeling MRI. Eur Radiol. 2016;26:244-53.

16. Chen $\mathrm{K}$, Langbaum JB, Fleisher AS, et al. Twelve-month metabolic declines in probable Alzheimer's disease and amnestic mild cognitive impairment assessed using an empirically pre-defined statistical region-of-interest: findings from the Alzheimer's Disease Neuroimaging Initiative. Neurolmage. 2010:51:654-64

17. Musiek ES, Chen Y, Korczykowski M, et al. Direct comparison of fluorodeoxyglucose positron emission tomography and arterial spin labeling magnetic resonance imaging in Alzheimer's disease. Alzheimers Dement. 2012;8:51-9.

18. Alsop DC, Detre JA, Golay X, et al. Recommended implementation of arterial spin-labeled perfusion MRI for clinical applications: a consensus of the ISMRM perfusion study group and the European consortium for ASL in dementia. Magn Reson Med. 2015;73:102-16.

19. Chen Y, Wolk DA, Reddin JS, et al. Voxel-level comparison of arterial spinlabeled perfusion MRI and FDG-PET in Alzheimer disease. Neurology. 2011; 77:1977-85.

20. Verclytte $\mathrm{S}$, Lopes $\mathrm{R}$, Lenfant $\mathrm{P}$, et al. Cerebral hypoperfusion and hypometabolism detected by arterial spin labeling MRI and FDG-PET in early-onset Alzheimer's disease. J Neuroimaging. 2016;26:207-12.

21. Binnewijzend MA, Kuijer JP, Benedictus MR, et al. Cerebral blood flow measured with 3D pseudocontinuous arterial spin-labeling MR imaging in Alzheimer disease and mild cognitive impairment: a marker for disease severity. Radiology. 2013;267:221-30.

22. McKhann G, Drachman D, Folstein M, Katzman R, Price D, Stadlan EM Clinical diagnosis of Alzheimer disease: report of the NINCDS-ADRDA Work Group under the auspices of Department of Health and Human Service Task Force on Alzheimer Disease. Neurology. 1984;34:939-44.

23. Folstein MF, Folstein SE, McHugh PR. "Mini-mental state". A practical method for grading the cognitive state of patients for the clinician. J Psychiatr Res. 1975;12:189-98.

24. Hatazawa J, lida H, Shimosegawa E, Sato T, Murakami M, Miura Y. Regional cerebral blood flow measurement with iodine-123-IMP autoradiography: normal values, reproducibility and sensitivity to hypoperfusion. J Nucl Med. 1997;38:1102-8

25. Pfefferbaum A, Chanraud S, Pitel A-L, et al. volumetric cerebral perfusion imaging in healthy adults: regional distribution, laterality, and repeatability of Pulsed Continuous Arterial Spin Labeling (PCASL). Psychiatry Res. 2010; 182:266-73

26. Jovicich J, Czanner S, Greve D, et al. Reliability in multi-site structural MRI studies: effects of gradient non-linearity correction on phantom and human data. Neurolmage. 2006;30:436-43.

27. Swan A, Waddell B, Holloway G, et al. The diagnostic utility of 99mTc-HMPAO SPECT imaging: a retrospective case series from a tertiary referral early-onset cognitive disorders clinic. Dement Geriatr Cogn Disord. 2015;39:186-93.

28. de Souza LC, Lehéricy S, Dubois B, Stella F, Sarazin M. Neuroimaging in dementias. Curr Opin Psychiatry. 2012;5:473-9.

29. Langbaum JB, Chen K, Lee W, et al. Categorical and correlational analyses of baseline fluorodeoxyglucose positron emission tomography images from 
the Alzheimer's Disease Neuroimaging Initiative (ADNI). Neurolmage. 2009; 45:1107-16.

30. Mosconi L. Brain glucose metabolism in the early and specific diagnosis of Alzheimer's disease. FDG-PET studies in MCl and AD. Eur J Nucl Med Mol Imaging. 2005;32:486-510.

31. Greicius MD, Srivastava G, Reiss AL, Menon V. Default-mode network activity distinguishes Alzheimer's disease from healthy aging: evidence from functional MRI. Proc Natl Acad Sci U S A. 2004;101:4637-42.

32. Hampel H, Prvulovic D, Teipel SJ, Bokde AL. Recent developments of functional magnetic resonance imaging research for drug development in Alzheimer's disease. Prog Neurobiol. 2011;95:570-8.

33. Buckner RL, Snyder AZ, Shannon BJ, et al. Molecular, structural, and functional characterization of Alzheimer's disease: evidence for a relationship between default activity, amyloid, and memory. J Neurosci. 2005:25:7709-17.

34. Damoiseaux JS, Prater KE, Miller BL, Greicius MD. Functional connectivity tracks clinical deterioration in Alzheimer's disease. Neurobiol Aging. 2012;33: 828.e19-30.

35. Liu Y, Zeng X, Wang Z, Zhang N, Fan D, Yuan H. Different post label delay cerebral blood flow measurements in patients with Alzheimer's disease using 3D arterial spin labeling. Magn Reson Imaging. 2015;33:1019-25.

36. Minoshima S, Frey KA, Koeppe RA, Foster NL, Kuhl DE. A diagnostic approach in Alzheimer's disease using three-dimensional stereotactic surface projections of fluorine-18-FDG PET. J Nucl Med. 1995;36:1238-48.

37. Matsuda H, Mizumura S, Nagao T, et al. Automated discrimination between very early Alzheimer disease and controls using an easy Z-score imaging system for multicenter brain perfusion single-photon emission tomography. AJNR Am J Neuroradiol. 2007;28:731-6.

38. Matsuda H, Mizumura S, Nagao T, et al. An easy Z-score imaging system for discrimination between very early Alzheimer's disease and controls using brain perfusion SPECT in a multicentre study. Nucl MedCommun. 2007;28: 199-205.

\section{Submit your next manuscript to BioMed Central and we will help you at every step:}

- We accept pre-submission inquiries

- Our selector tool helps you to find the most relevant journal

- We provide round the clock customer support

- Convenient online submission

- Thorough peer review

- Inclusion in PubMed and all major indexing services

- Maximum visibility for your research

Submit your manuscript at www.biomedcentral.com/submit 